\title{
Voice over Internet Protocol (VoIP) Based IP PBX System Design
}

\author{
Sonali Golhar ${ }^{1}$, V. S. Dhamdhere ${ }^{2}$ \\ ${ }^{1}$ Computer Department, G. H. Raisoni COEM, Wagholi, Pune-412207, Maharashtra, India \\ ${ }^{2}$ Professor, Computer Department, G. H. Raisoni COEM, Wagholi, Pune-412207, Maharashtra, India
}

\begin{abstract}
Voice over internet Protocol (VoIP) technologies are integral to trendy telecommunications attributable to their advanced options, flexibility, and economic advantages. web Service suppliers at first promoted these technologies by providing low value native and international occupation. At present, there's additionally a good deal of interest in mistreatment IP-based technologies to switch ancient little and enormous workplace telephone systems that use ancient PBX's (Private Branch eXchange). sadly, the massive majority of the rising VoIP based mostly workplace telephone systems have followed the centralized design of ancient public and personal telephone systems within which all the intelligence within the system is at the core, with quite expensive hardware and code parts and applicable redundancy for adequate levels of responsibility. The paper is meant to explain and analyze the feasibleness of the results of the VoIP system designed if enforced during a field network like national capital University telephone network. so the convergence strategy of an IP PABX system with the present wire telephone network has been mentioned. whereas taking this into thought, upgrading technique of the prevailing system using trendy network appliances has been delineate and at a similar time new configuration connecting this science network with the telephone network has been designed. This study entails the simulation and implementation of a VoIP telecom system mistreatment an IP PBX resolution. a replacement technology known as voice over internet Protocol (VoIP), or web telecom means your voice is carried over the IP network, otherwise referred to as the net. Voice, that is an analogue signal, is converted to digital knowledge, that is then disassembled and transmitted through net|the net\} only to be reconverted back to an analogue signal on the opposite finish using an IP-PBX resolution that may be a UNIX base server that supports a interface web interface. This service may be properly managed and deployed over a network with less stress and expenses.
\end{abstract}

Keywords: VoIP, Asterisk, PBX, IAX, SIP, Trunks

\section{Introduction}

Voice over internet Protocol (VoIP) may be a varied topic. there's no single templet which will be followed once considering the utilization of this technology to supersede a standard telephone system. The technology itself is fairly generic and somewhat mature, but the impact to the external atmosphere, business conditions close the particular necessities, capital funding arrange and different integral factors complicate the analysis. Implementing VoIP at Dhaka University, is way constant as elsewhere apart from distinctive characteristics endemic to the University. it's but, these distinctive characteristics that warrant a better look since capital investment are going to be important and once an answer is put in, it'll presumably stay long on the far side its' meant helpful life.

Within the range of the University, there are many factors that differentiate this environment from that of a company one. though these don't have an effect on the technology trend or the economics related to VoIP readying, they are doing influence the character and sort of readying field wide. This document highlights several key areas that require to be self-addressed once considering large-scale readying of VoIP likewise as some special issues relative to the University[5]. The results aren't stunning once all influences are thoughtabout. If we tend to analyze the business want then think about the acceptable variables, the strategy appears simple. a number of the variables to think about are value, the present infrastructure, implementation practicality, maintenance, operability and therefore the University topology.
The structure of the wire line telephone network has not essentially modified since Alexander Graham Bell fictitious the telephone 130 years past. Calls move dedicated phone lines and are switched in numerous places to keep up an electronic circuit leading from the phone wherever the decision is created to the phone with the quantity being known as thence the phrase -ircuit-switched network" [1].While digital technologies have exaggerated the practicality of the telephone system, any intelligence within the system is basically built into the circuit switches of the phonephone company's headquarters. distinction thereto the broadband net world. customers use a laptop to sort an email that's then separated into digital packets (groups of ones and zeros) that are transported via a broadband -pipe" onto highspeed backbone networks. The packets are then reassembled at the destination account, which may be designed for access from any net connected computer anyplace within the world [7]. a few years past it absolutely was discovered that causing a symbol to a far off destination may have be done conjointly in a digital fashion: before causing it we've to digitalize it with an ADC (analog to digital converter), transmit it, and at the top rework it once more in analog format with DAC (digital to analog converter) to use it. $\checkmark$ OIP works like that, digitalizing voice in information packets, causing them and reconverting them in voice at destination". Digital format will be higher controlled: we will compress it, route it, convert it to a replacement higher format, and then on; conjointly we tend to saw that digital signal is additional noise tolerant than the analog one (GSM vs. TACS) [3]. TCP/IP networks are product of information processing packets containing a header (to management 


\section{International Journal of Science and Research (IJSR) \\ ISSN (Online): 2319-7064}

Index Copernicus Value (2013): 6.14 | Impact Factor (2014): 5.611

communication) and a payload to move data: VOIP uses it to travel across the network and are available to destination.

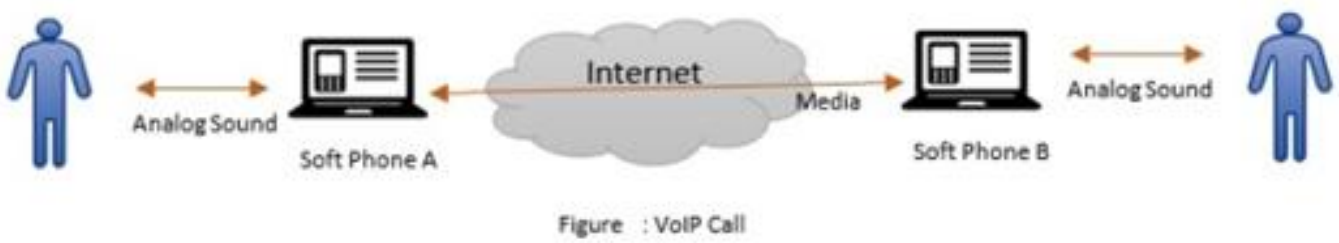

Voice over information processing (VoIP) may be a methodology and cluster of technologies for the delivery of voice communications and multimedia system sessions over internet Protocol (IP) networks, like the web. Different terms unremarkably related to VoIP are IP telecom, net telecommunication, broadband telephony, and broadband phone service.

The term net telecommunication specifically refers to the provisioning of communications services (voice, fax, SMS, voice-messaging) over the general public net, instead of via the general public switched telephone network (PSTN). The steps and principles concerned in originating VoIP telephone calls are the same as ancient digital telecom and involve communication, channel setup, conversion of the analog voice signals, and secret writing. Rather than being transmitted over a circuit-switched network, however, the digital info is packetized, and transmission happens as information processing packets over a packet-switched network [9]. Such transmission entails careful issues regarding resource management completely different from time-division multiplexing (TDM) networks.

Early suppliers of voice-over-IP services offered business models and technical solutions that reflected the design of the legacy telephone network. Second-generation providers, like Skype, have designed closed networks for personal user bases, providing the good thing about free calls and convenience whereas doubtless charging for access to different communication networks, like the PSTN. This has restricted the liberty of users to mix-and-match third-party hardware and package. Third-generation suppliers, like Google speak, have adopted the thought of federate VoIPwhich may be a departure from the design of the legacy networks. These solutions generally enable dynamic interconnection between users on any 2 domains on the web once a user needs to position a decision.

VoIP systems use session management and communication protocols to manage the communication, set-up, and teardown of calls. They transport audio streams over IP networks using special media delivery protocols that encrypt voice, audio, video with audio codecs, and video codecs as Digital audio by streaming media [11]. Numerous codecs exist that optimize the media stream supported application necessities and network bandwidth; some implementations place confidence in narrowband and compressed speech, whereas others support high fidelity stereo codecs. Some widespread codecs embody $\mu$-law and a-law versions of G.711, G.722, a well-liked open supply voice codec referred to as iLBC, a codec that only uses 8kbit/s every method known as G.729, and plenty of others.

\section{Literature Survey}

J. Rosenberg, H. Schulzrinne, G. Camarillo,A. Johnston, J. Peterson,R. Sparks, M. Handley,and E. Schooler,[2]This document describes Session Initiation Protocol (SIP), associate application-layer management (signaling) protocol for making, modifying, and terminating sessions with one or a lot of participants. These sessions embody web telephone calls, multimedia system distribution, and multimedia system conferences. SIP invites wont to produce sessions carry session descriptions that permit participants to agree on a group of compatible media varieties.SIP makes use of parts known as proxy servers to assist route requests to the user's current location, authenticate and authorize users for services, implement supplier call-routing policies, and supply options to users. SIP conjointly provides a registration perform that permits users to transfer their current locations to be used by proxy servers. SIP runs on prime of many completely different transport protocols.

H. Schulzrinne and S. Casner,[1]This note describes a profile for the utilization of the period of time transport protocol (RTP), version 2, and therefore the associated management protocol, RTCP, among audio and video multi participant conferences with marginal management. It provides interpretations of generic fields among the RTP specification appropriate for audio and video conferences. Particularly, this document defines a group of default mappings from payload sort numbers to encodings. The document conjointly describes however audio and video knowledge could also be carried among RTP. It defines a group of ordinary encodings and their names once used among RTP. However, the encryption definitions are freelance of the actual transport mechanism used. The descriptions give tips to reference implementations and therefore the careful standards. This document is supposed as associate aid for implementers of audio, video and alternative period of time multimedia system applications.

M. Handley, V. Jacobson, and C. Perkins, [12] Forte Skype could be a peer-to-peer VoIP consumer developed by KaZaa in 2003. Skype claims that it will work nearly seamlessly across NATs and firewalls and has higher voice quality than the MSN and Yahoo IM applications. It encrypts calls endto-end, and stores user info in a much localized fashion. Skype conjointly supports instant messaging and conferencing. This report analyzes key Skype functions like login, NAT and firewall traversal, decision institution, media transfer, codecs, and conferencing under 3 completely different network setups. Analysis is performed by careful study of Skype network traffic.

D. Adami, C. Callegari, S. Giordano, M. Pagano, and T. Pepe,[7] This paper highlights the planning and implementation aspects of a VoIP primarily based Asterisk 


\section{International Journal of Science and Research (IJSR) \\ ISSN (Online): 2319-7064 \\ Index Copernicus Value (2013): 6.14 | Impact Factor (2014): 5.611}

voice exchange, developing a completely practical voice exchange needs to line up a server supported Asterisk, connecting clients thereto server with the assistance of soft phones then configuring the softphones with the server. Here in our implementation we've got connected the purchasers to the server with the assistance of IAX protocols. The primary a part of the paper contains some introductory ideas regarding VoIP, followed by Asterisk's internal design. within the third a part of the paper we tend to discuss regarding the codec's and protocols employed by the packet switching primarily based PBX networks and eventually we tend to brush up regarding the planning and implementation aspects.

H. J. Kang, Z.-L. Zhang, S. Ranjan, and A. Unci, [9]with the widespread adoption of SIP-based VoIP, understanding the characteristics of SIP traffic behavior is vital to downside designation and security protection of VoIP services - two key aspects of providing dependable VoIP services. During this paper we tend to propose a general methodology for identification SIP-based VoIP traffic behavior at many levels: SIP server host, server entity (e.g., registrar and call proxy) and individual user levels - to derive -normal" behavior profiles. Using SIP traffic traces captured ina production VoIP network, we tend to illustrate the characteristics of SIP-based VoIP traffic behavior in associate operational surroundings and demonstrate the effectiveness of our general identification methodology. Primarily based upon the identification methodology, we tend to develop an easy and yet effective entropy-based anomaly detection formula for detection potential security attacks furthermore as performance issues. We tend to demonstrate the efficacy of our formula in detection potential VoIP attacks through work experimentation.

\section{Proposed Approach Framework and Design}

\section{Architecture}

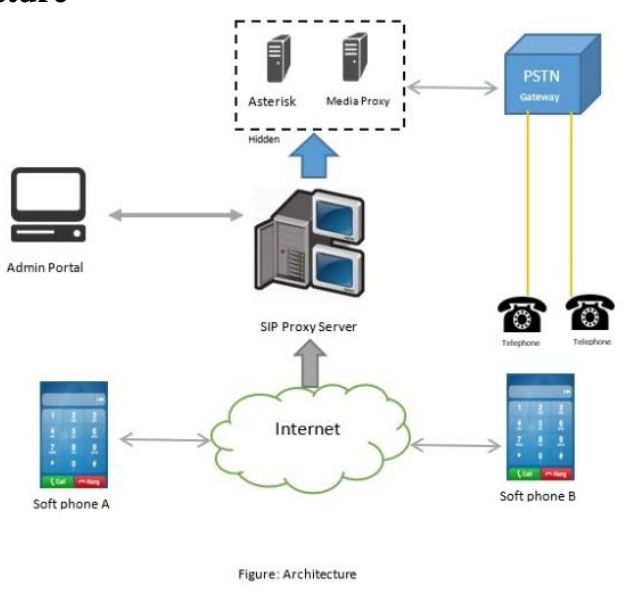

Figure: Proposed System Architecture

\section{Process Flow}

Asterisk: may be a software implementation of a telephone private branch exchange (PBX); it permits connected telephones to form calls to at least one another, and to attach to alternative telephone services, like the public switched telephone network (PSTN) and voice over internet Protocol (VoIP) services. Its name comes from the asterisk image, *.
Asterisk is free with a twin license model, using the gnu General Public License (GPL) as a free software license and a proprietary software license to allow licensees to distribute proprietary, unpublished system parts.

SIP: The Session Initiation Protocol (SIP) may be a rule for communication and dominant transmission communication sessions. The foremost common applications of SIP are in internet telecommunication for voice and video calls, likewise as instant messaging, over internet Protocol (IP) networks.

The protocol defines the messages that are sent betweenendpoints that govern institution, termination and alternative essential parts of a decision. SIP may be used for making, modifying and terminating sessions consisting of 1 or many media streams. SIP is associate application layer protocol designed to be freelance of the underlying transport layer. It's a text-based protocol, incorporating several parts of the hypertext Transfer Protocol (HTTP) and therefore the easy Mail Transfer Protocol (SMTP).

We handled the identification of VoIP traffic in UDP Flows The heuristics to identify RTP or RTCP in UDP packets depends on their documentations, principally the RFCs. These heuristics uses information contained at intervals the mounted packet headers.

Above mentioned parts is that the most vital a part of bestowed Project. We've utilised the fullest of the mentioned parts to induce the higher result.

\section{Mathematical Model}

A typical voice packet would pass through several nodes before arriving at the destination. We consider the voice traffic served by a single server as well as multiple server a server effectively constitutes a node of the network. Two or more server is connected by transmission lines.

\section{Threshold Delay, Resource Consumption and Throughput}

For an $\mathrm{M} / \mathrm{M} / 1$ system, the probability that a voice packet suffers a delay less than $t$ is given by $[58,81]$,

$$
\rho_{1}=\rho\left\{\mathrm{w}_{1} \leq \mathrm{t}\right\}=1-\rho \mathrm{e}^{-\mu \mathrm{C}}(1-\mathrm{p}) \mathrm{t} .
$$

Therefore, the throughput of the single -hop VoIP network where all packets that undergo a queuing delay not exceeding the threshold delay can be expressed as:

$$
\gamma_{1}=\lambda \rho_{1}
$$

Where $\lambda$ is the arrival rate of packets?

The normalized throughput, i.e. throughput expressed as a function of the incident traffic can be given as:

$$
\rho_{1}=\frac{v_{1}}{\lambda}
$$

$\rho=$ traffic intensity

$\mathrm{t}=$ threshold delay 
$\mathrm{C}_{1}=$ capacity

$\rho_{1}=$ parameter

$\lambda=($ Given value $)$

$\mu=$ (given value)

$\lambda=$ given 2000

$\mu$ = given 1

The relative gain in normalized throughput reduces as the threshold delay is increased. Even as the normalized throughput monotonically increases. It would be interesting to determine if the resulting throughput per unit of bandwidth used shows to determine if the resulting throughput per unit of bandwidth used shows a point of inflxion

From Eqs.7.1 and 7.2, we have,

$$
\frac{\gamma_{1}}{C_{1}}=\frac{\lambda}{C_{1}}\left[1-\frac{\lambda}{\mu C_{1}} e^{-\left(\mu C_{1-\lambda}\right) t}\right]
$$

A point of inflexion can be determined by putting

$$
\frac{d\left(\gamma_{1} / C_{1}\right)}{d C_{1}}=0
$$

Which result in

$$
\mu C_{1 \mathrm{opt}} \mathrm{e}^{(} \pi C_{1 \mathrm{opt}}-\lambda^{\mathrm{t}}=\lambda\left(2+\mu \mathrm{C}_{1 \mathrm{opt}}^{\mathrm{t}}\right)
$$

Where $\mathrm{C}_{1 \mathrm{opt}}$ is the optimum capacity needed for the system to reach the Maximum throughput per unit of bandwidth. Equation 7.4 shows a maximum value because

$$
\frac{d^{2}\left(\gamma_{1} / C_{1}\right)}{d C_{1}^{2}}=\frac{2 \lambda}{C_{1}^{3}}\left\{1-\frac{\lambda}{2 \mu C_{1}}\left[\left(\mu C_{1 \mathrm{t}}+2\right)^{2}+2\right] \mathrm{e}^{-(} \pi c_{1}^{-\lambda) \mathrm{t}}\right\}
$$

Is negative.

\section{Work Done}

In this section we are discussing the practical environment, scenarios, performance metrics used etc.

Hardware Requirements:

$\begin{array}{lc}\text { Processor } & : \text { Pentium IV 2.6 GHz } \\ \text { Ram } & : 512 \mathrm{Mb}\end{array}$

Hard Disk $\quad$ : $20 \mathrm{~GB}$

Software Requirements:

$\begin{array}{ll}\text { Front End } & : \text { J2SE } \\ \text { Back End } & \text { : MySql 5.1 } \\ \text { Tools Used } & : \text { Eclipse/Nebeans } \\ \text { Operating System: } & \text { Windows 7/8 }\end{array}$

\section{Results of Practical Work}

Now after creating the extensions now we configure the Softphone on the client/user system which might be windows, Linux or Mac. The software is X-lite/Blink which is available on www.counterpath.com. Buy and download the software and install it. Now by successful installation the extension which is created in server is now configured here by entering the details in SIP proxy setting.

Display name: Harry

SIP address: 1234@sip2sip.info

Password:

Once we have all the options set, close the windows and $\mathrm{X}$ lite/Blink will attempt to connect to our server. If everything is set properly, X-lite/Blinkwill tell us that we are logged in and display our extension number.

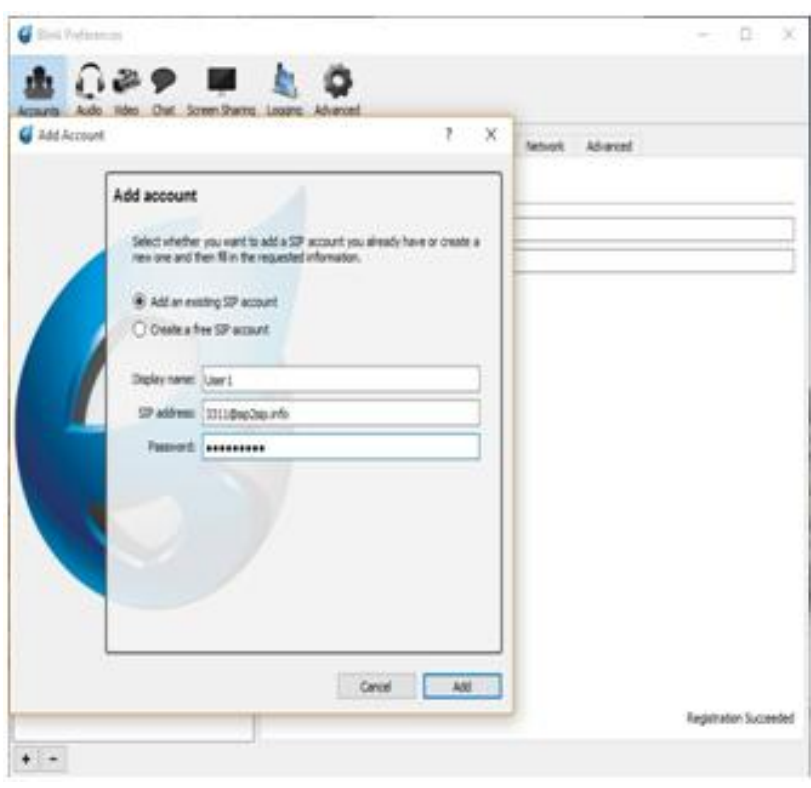

Figure: Softphone Configuration(a)

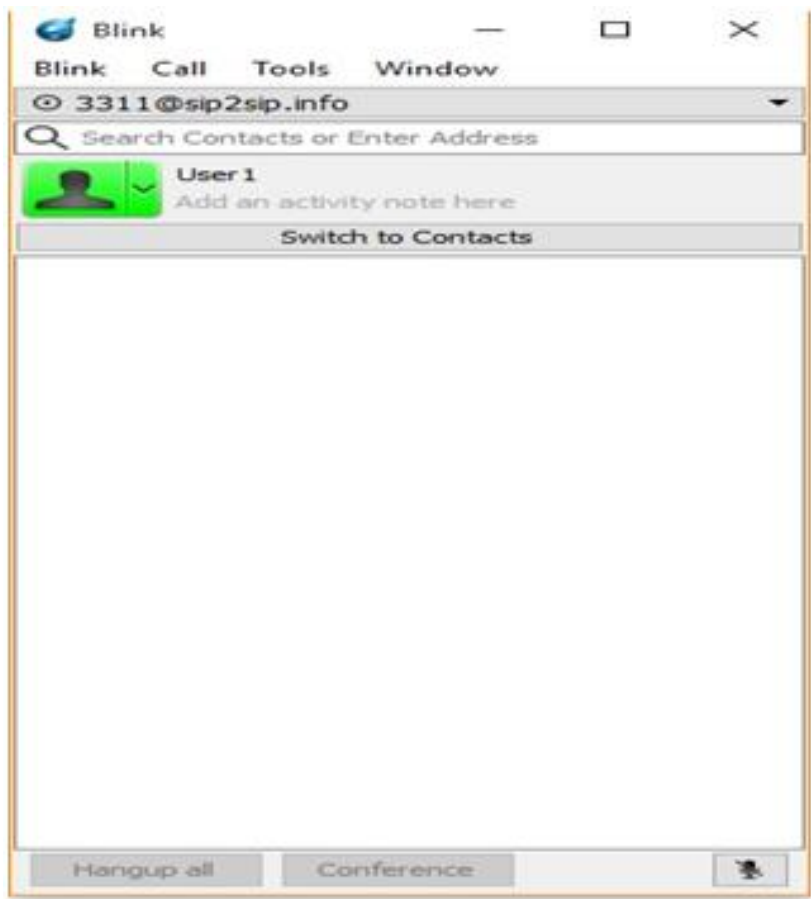

Figure: Softphone Configuration(b)

If our phone has successfully registered with Asterisk PBX, type in *65 and hit Enter. This will tell Asterisk to read back our current extension. If we hear the voice say Your extension is 100 ", then we are well on our way to getting our system fully operational.

If everything is working, we can set up a second extension and make sure the two extensions can call each other and that both sides can hear audio from the other side. We now have a working telephone system with extensions that can call each other. 


\section{International Journal of Science and Research (IJSR) \\ ISSN (Online): 2319-7064}

Index Copernicus Value (2013): 6.14 | Impact Factor (2014): 5.611
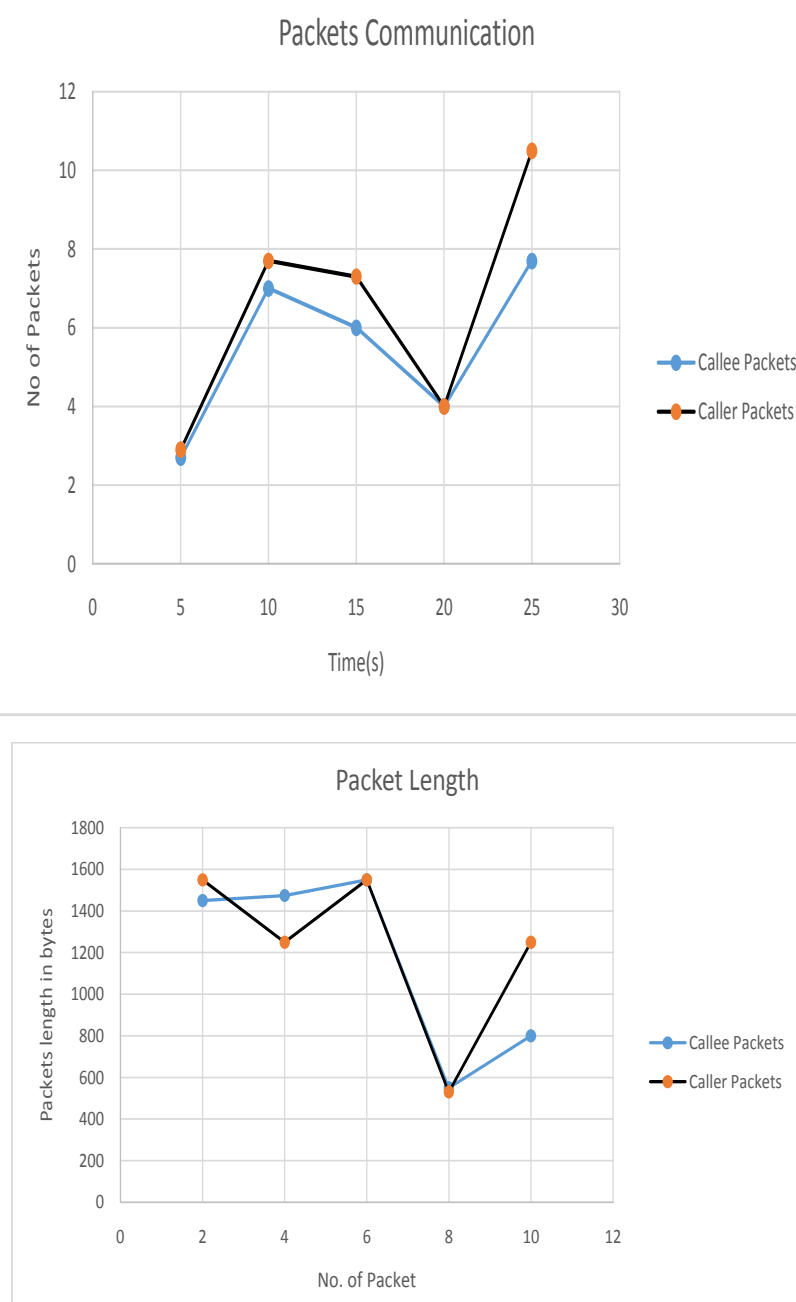

Packet Comparison

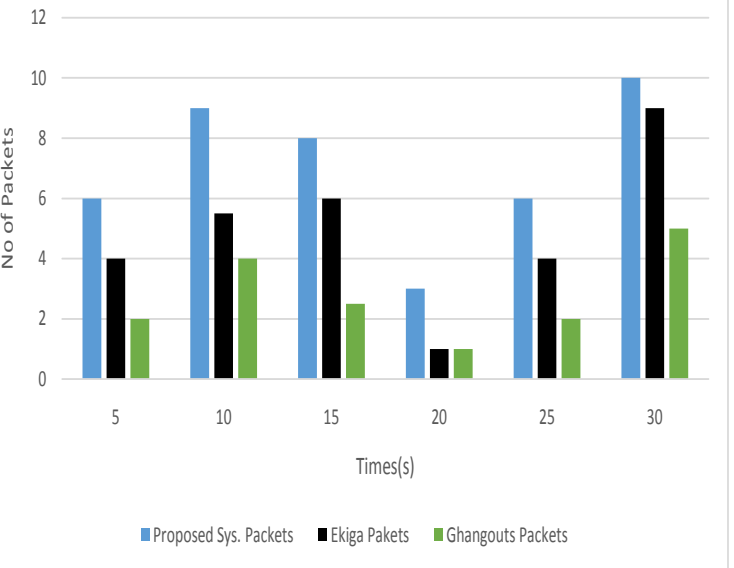

\section{Conclusion and Future Work}

If the late 1990s were characterised by irrational (or a minimum of premature) exuberance for the innovative promise of the IT revolution, the previous few years are characterised by the other trend: an irrational pessimism regarding the semipermanent edges of IT and VIOP specially. As public attitudes choose a a lot of realistic middle ground and new innovations spur growth, one driver of continued progress are VOIP. VoIP isn't free, or price effective all told cases. it's most effective in plain vanilla cases once traditional systems are displaced for a range of reasons, together with devolution or high maintenance price. At the University, we have a tendency to ar within the throes of attempting to ascertain unified voice design, whereas leverage viable assets in order that we tend to might attain a technologically sound answer, that is additionally economically possible. This paper provides info regarding the IPPBX and its communication. The reliableness of the system is totally depends upon the network. If the network fails the complete communication fails. additionally to speak with outside the organization the net facility should be out there and thus, the net charges are applicable otherwise forthe internal communication the business are freed from charge. we tend to handled the identification of VoIP traffic in UDP Flows. The heuristics to identify RTP or RTCP in UDP packets depends on their documentations, principally the RFCs. These heuristics uses information contained among the mounted packet headers.

\section{References}

[1] H. Schulzrinne, S. Casner, R. Frederick, and V. Jacobson, RFC3550: RTP: A Transport Protocol for Real-Time Applications," IETF,Tech. Rep., 2003, http://tools.ietf.org/html/rfc3550.[Online].Available:w ww.ietf.org/rfc/rfc3550.txt

[2] J. Rosenberg, H. Schulzrinne, G. Camarillo, A. Johnston, J. Peterson,R. Sparks, M. Handley, and E. Schooler, RFC 3261: SIP: SessionInitiation Protocol," IETF, $\quad$ Tech. 2002. [Online].Available:www.ietf.org/rfc/rfc3261.txt

[3] Fun with xmpp and google talk," http://www.adarshr.com/papers/xmpp.

[4] Jingle," http://xmpp.org/extensions/xep-0166.html.

[5] Jingle rtp sessions," http://xmpp.org/extensions/xep0167.html.

[6] S. Moln'ar and M. Per'enyi, -Orthe identification and analysis of skype traffic," Int. J. Commun. Syst., vol. 24, no. 1, pp. 94-117, Jan. 2011.[Online]. Available: http://dx.doi.org/10.1002/dac.1142

[7] D. Adami, C. Callegari, S. Giordano, M. Pagano, and T. Pepe, Skypehunter:A real-time system for the detection and classification of skypetraffic," Int. J. Communication Systems, vol. 25, no. 3, pp. 386403,2012.

[8] -Skype: A Practical Security Analysis," SANS, Tech. Rep. [Online].Available: http://www.sans.org/reading room/whitepapers/voip/32918.php

[9] H. J. Kang, Z.-L. Zhang, S. Ranjan, and A. Nucci, Sip-based voip traffic behavior profiling and its applications," in Proceedings of the3rd annual ACM workshop on Mining network data, ser. MineNet '07.New York, NY, USA: ACM, 2007, pp. 39-44. [Online].

Available:http://doi.acm.org/10.1145/1269880.126989 1

[10] T. Sinam, I. T. Singh, P. Lamabam, and N. Ngasham, -Anefficient technique for detecting skype flows in udp media streams," in Proc.IEEE International Conference on Advanced Networks and Telecommunication Systems (IEEE ANTS), Chennai, India, Dec 2013. 


\section{International Journal of Science and Research (IJSR) \\ ISSN (Online): 2319-7064}

Index Copernicus Value (2013): 6.14 | Impact Factor (2014): 5.611

[11] H. Schulzrinne and S. Casner, RTP Profile for Audio and Video Conferences with Minimal Control," RFC 3551

(Standard), 2003,http://www.ietf.org/rfc/rfc3551.txt.

[12] M. Handley, V. Jacobson, and C. Perkins, -SDP Session Description Protocol," RFC 4566 (Proposed Standard), Internet Engineering TaskForce, Jul. 2006. [Online]. Available: http://www.ietf.org/rfc/rfc4566.txt

[13] D. Bonfiglio, M. Mellia, M. Meo, D. Rossi, and P. Tofanelli, Revealing skype traffic: when randomness plays with you," SIGCOMM Comput.Commun. Rev., vol. 37, no. 4, pp. 37-48, Aug. 2007. [Online].Available:

http://doi.acm.org/10.1145/1282427.1282386

[14] H. Wang, Skype voip service-architecture and comparison," in INFOTECH Seminar Advanced Communication Services (ASC), 2005,p. 4.

[15] S. Baset and H. Schulzrinne, -Aranalysis of the skype peer-to-peer internet telephony protocol," in INFOCOM, 2006.

[16] J.-L. Costeux, F. Guyard, and A.-M. Bustos, Đetection and comparison of rtp and skype traffic and performance." in GLOBECOM. IEEE, 2006. [Online]. Available:http://dblp.unitrier.de/db/conf/globecom/glob ecom2006qrp.html\#CosteuxGB06

[17] S. Arena, R. Birke, M. Mellia, M. Petracca, C. Racca, and D. Rossi,-Multimedia and streaming traffic analysis," 2006, 3rd EuroNGI Workshopon New Trends in Modelling, Quantitative Methods and Measurements, Torino, Italy 22-23 June 2006.

[18] Wireshark," http://www.wireshark.org/.

[19] rtptools,"http://www.cs.columbia.edu/IRT/software/rt ptools/.

[20] -7-filter," http://17-filter.sourceforge.net/protocols.

[21] M. Erdal and K. Hedayat, -Real-time transport protocol stream detection system and method," Patent US 8306 063, 11 06, 2012.[Online]. Available: http://www.patentlens.net/patentlens/patent/US\830606 3/en/ 